\title{
A virada das mobilidades: fluxos, fixos e fricções
}

The Mobilities Turn: Flows, Fixities and Frictions

Le tournant des mobilités : fluxes, fixités et frictions

\section{Bianca Freire-Medeiros e Mauricio Piatti Lages}

\section{OpenEdition}

\section{Journals}

Edição electrónica

URL: http://journals.openedition.org/rccs/11193

DOI: $10.4000 /$ rccs. 11193

ISSN: 2182-7435

\section{Editora}

Centro de Estudos Sociais da Universidade de Coimbra

\section{Edição impressa}

Data de publição: 1 dezembro 2020

Paginação: 121-142

ISSN: 0254-1106

\section{Refêrencia eletrónica}

Bianca Freire-Medeiros e Mauricio Piatti Lages, «A virada das mobilidades: fluxos, fixos e fricções », Revista Crítica de Ciências Sociais [Online], 123 | 2020, posto online no dia 15 dezembro 2020, consultado o 17 dezembro 2020. URL : http://journals.openedition.org/rccs/11193 ; DOI : https:// doi.org/10.4000/rccs. 11193

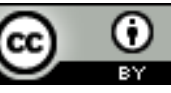




\section{BIANCA FREIRE-MEDEIROS, MAURICIO PIATTI LAGES}

\section{A virada das mobilidades: fluxos, fixos e fricções*}

Pelo menos desde os anos 2000, testemunhamos o esforço, em diferentes campos disciplinares e a partir de realidades empíricas diversas, para compreender a complexidade do movimento interdependente de pessoas e capitais, bens e imagens. Mas como evitar, na negação de categorias estáticas e metodologias sedentárias, a substituição do que é próprio do Estado-nação pela imprecisão conceitual dos "líquidos" ou pela romantização do nomadismo? Como incorporar, à discussão sobre a mobilidade, as práticas regulatórias e as desigualdades que a estruturam? Qual seria, em resumo, o estatuto teórico das mobilidades - e imobilidades - na teoria social? Este artigo descreve algumas das soluções analíticas propostas pela "virada das mobilidades", tomando como fio condutor as contribuições daquele que é reconhecido como seu principal articulador, o sociólogo britânico John Urry.

Palavras-chave: epistemologia das ciências sociais; mudança epistêmica; teoria social.

\section{Introdução}

A partir dos anos 1980, as ciências sociais se viram desafiadas a refletir sobre os processos de reestruturação econômica, de compressão espaço-temporal, de expansão dos mercados de bens culturais, de migração de "sujeitos coloniais" rumo às sedes dos antigos impérios, de crescente dependência dos fluxos digitais e de aceleração da vida cotidiana identificados sob a alcunha de globalização (cf. King, 1984; Harvey, 1992; Ortiz, 1994; Santos, 2001). A formulação de diagnósticos sobre esses fenômenos de extensão global implicou a reconfiguração das noções de "cultura", "sociedade", "identidade" e "Estado-nação", ainda tomadas como autoevidentes. Passaram a

\footnotetext{
Este artigo é devedor do diálogo crítico com Alexandre Magalhães, Apoena Mano, Camila Moraes, Diego Peralta, João Freire Filho, João Freitas, Maria Alice Nogueira, Nathalia Silva e Palloma Menezes. A cada um/uma deixamos nosso agradecimento público que, obviamente, os/as isenta de todas as imprecisões cometidas por nós.

Parte deste artigo foi desenvolvido no âmbito do doutorado de Mauricio Piatti Lages, financiado pela Fundação de Amparo à Pesquisa do Estado de São Paulo (FAPESP), processo n. ${ }^{\circ} 2016 / 24696-2$.
} 
proliferar metáforas cujo fim último seria descrever e analisar um mundo social on the move: rede (network, web, mesh), fluxo (flow, flux), líquido (liquid), fluido (fluid), espacialidade (spatiality), paisagem (scape), viagem (travel), corrente (stream), rota (route, path).

Mas como operacionalizar uma compreensão dos processos contemporâneos - da transnacionalização dos mercados financeiros e de commodities à "dissolução das identidades sociais" (Hall, 1997), passando pela ascensão das ditas cidades globais (King, 1984; Sassen, 1991) - sem cair na romantização da fluidez e do nomadismo? Como evitar, na negação de categorias estáticas e metodologias sedentárias, a substituição do que é próprio ao repertório cognitivo e político do "nacionalismo metodológico" (Beck, 2002; Wimmer e Glick Schiller, 2002; ver também Elias, 1994) pelo fetichismo do movimento? Qual seria o estatuto teórico e o potencial heurístico das mobilidades - e das imobilidades - como articulador analítico na teoria social?

Essas indagações animaram a "virada das mobilidades", guinada epistêmica institucionalizada no início do século XXI, mas cuja origem remete às teorizações que levaram ao spatial turn dos anos 1980 (Gregory e Urry, 1985; ver Sheller e Urry, 2006 e Sheller, 2014, 2017 para um mapeamento do contexto institucional e de outras correntes de influência). Antes tido como apartado do "social" e preterido em nome de um tempo concebido como domínio ativo das transformações humanas, o espaço assume uma centralidade inédita até então. Já não se tratava, porém, do espaço enraizado da tradição etnográfica - o campo territorialmente circunscrito e ontologicamente estável -, mas da observação das rotas pelas quais as formas socioculturais são feitas e refeitas (Clifford, 1997), de conjuntos de relações multilocalizadas (Marcus, 1995). Partindo dessas propostas, a virada das mobilidades busca evitar o "fetichismo espacial" das noções mais substancialistas da sociedade como território fixo ou autocontido, redirecionando o foco para as espacializações móveis.

Nem apenas metáfora, nem redutível a referentes empíricos, as mobilidades constituem, no mobilities turn, uma perspectiva analítica a partir da qual se pode estudar qualquer fenômeno em que haja o entrelaçamento de movimento, representações e práticas, sustentado por infraestruturas materiais conectadas (Cresswell, 2006; Hannam et al., 2006). Desde diferentes matrizes disciplinares, quem se identifica com essa proposta atribui centralidade às linhas que conectam os pontos e dão complexidade ao movimento - realizado ou impedido, mas sempre regulado por normas e saberes - de pessoas e capitais, bens e imagens, informações e tecnologias, riscos e serviços.

As escalas de observação ativadas pelas lentes das mobilidades também são múltiplas: desde o nível das microinterações cotidianas, onde há corpos racializados, generificados e patologizados, buscando mover-se ou fixar-se; 
passando por um mesonível de questões ligadas à escala do urbano e das instituições regionais; chegando ao macronível das relações transnacionais (Sheller, 2018). Além disso, o fato de haver uma sobrerrepresentação de pesquisas voltadas para o tempo presente não significa ausência de empreendimentos de investigação histórica bem-sucedidos (ver Revill, 2014), assim como a preponderância de publicações em inglês não anula a relevância das pesquisas realizadas em outros contextos linguísticos (cf. Singh et al., 2017; Freire-Medeiros et al., 2018; Nogueira e Moraes, 2020).

Ainda que, como operador analítico-metodológico, a mobilidade não diga respeito a um objeto ou a uma escala específicos e, tampouco, seja de domínio exclusivo de uma corrente teórica ou de um campo disciplinar, privilegiamos o giro implementado pelo new mobilities paradigm (NMP), que encontra suas principais proposições de síntese na chamada Escola de Lancaster. Recuperamos, assim, algumas das soluções analíticas propostas pelo grupo multidisciplinar que orbita em torno do Center for Mobilities Research (CeMoRe), ${ }^{1}$ tomando como fio condutor as contribuições daquele que é reconhecido como seu principal articulador e um dos nomes mais influentes da sociologia britânica, Distinguished Professor da Universidade de Lancaster, John Urry (1946-2016).

Não há sentido em reduzir a virada das mobilidades a uma linha evolutiva ou a um punhado de marcos cognitivos. Para clareza da exposição, porém, recorremos ao tripé que se faz presente no subtítulo deste artigo: fluxos, fixos e fricções. Partimos da ideia de capitalismo desorganizado, formulada no início dos anos 1990, para examinar o deslocamento epistêmico provocado pela proposta de uma sociologia que, diante da obsolescência da própria ideia de "sociedade", volta-se para redes e fluxos. Na seção seguinte, recuperamos, em linhas gerais, o diálogo de Urry com as ciências duras, que o leva à concepção dos sistemas complexos em rede, destacando o problema - talvez o mais robusto, do ponto de vista analítico - da interseção sistêmica entre os diferentes tipos de mobilidades e sua dependência dos fixos infraestruturais. As fricções revelam-se em sua potência desestabilizadora na seção três, na qual demonstramos como a revisão crítica da ideia de rede e a ampliação do conceito de capital, empreendida pelo NMP, pautam o tema das desigualdades em novos termos. Buscamos, ainda, mostrar como a noção de regime de mobilidades pode estimular a reflexão necessária sobre a mobilidade como recurso, valor e direito, ao propor o exame das práticas regulatórias e governamentais que a estruturam e definem.

\footnotetext{
${ }^{1}$ Principal núcleo de propagação do NMP. Ver https://www.lancaster.ac.uk/cemore/aboutcemore/, consultado a 18.02.2020.
} 
Estamos cientes que privilegiar um autor, como fazemos aqui, deixa à sombra tanto os debates internos e críticas extrínsecas que atravessam o giro móvel, quanto a riqueza dos protocolos metodológicos que perfazem os métodos móveis (ver Buscher et al., 2011). O artigo deve ser lido, portanto, como um roteiro de temas do NMP por via da vastíssima obra de John Urry, cujo modelo analítico, concebido no redemoinho das mobilidades, foi ele mesmo mutante. Não por acaso, em seus últimos anos de vida, Urry dedicou grande energia intelectual e política à formulação de uma sociologia dos futuros possíveis - e desejáveis. ${ }^{2}$ Inspirados por essa postura, nas considerações finais permitimo-nos refletir sobre a redefinição da "boa vida móvel" que a pandemia de covid-19 está provocando em escala transnacional. Rascunhadas no calor dos acontecimentos, essas reflexões têm o intuito de trazer à tona, através de alguns nexos empíricos, uma problematização tanto da ideia de distância quanto dos discursos acerca da globalização pautados na mobilidade irrestrita de pessoas, coisas e signos.

\section{Metáforas para um "capitalismo desorganizado"}

Em The End of Organized Capitalism, Scott Lash e John Urry (1987) sugerem uma periodização tripartida dos momentos de inflexão nos modos de vida das sociedades ocidentais: entre o início da Revolução Industrial e o fin-de-siècle, teríamos o "capitalismo liberal"; até fins da década de 1960, o que os autores qualificam como "capitalismo organizado"; desde então, estaríamos sob a égide de um "capitalismo desorganizado" - o que adiante chamarão de "economias de signos e espaço", título do segundo livro da dupla (Lash e Urry, 1994). Como condição de possibilidade de emergência dessa nova economia, marcada pela expansão intersetorial e transnacional da produção e do consumo, estaria o incremento da mobilidade do capital. Agora integrado em redes globalizadas e dependentes de sistemas computadorizados cada vez mais abrangentes, ágeis e complexos, os fluxos monetários passam a ser garantidos pela desregulamentação dos quadros jurídico-normativos dos sistemas financeiros nacionais. Lash e Urry sublinham, e isso é fundamental para o argumento do NMP, que a intensificação dos fluxos seria impensável sem "estruturas de informação e comunicação transnacionais": agentes sociotécnicos, organizações públicas e privadas, todos conectados graças a uma série de "links" físicos (do avião aos cabos de fibra óptica) e virtuais (com destaque para a Internet).

Face a esse novo arranjo, já não seria produtivo dar centralidade às metáforas forjadas para interpretar o ordenamento contido por fronteiras

\footnotetext{
${ }^{2}$ Ver https://www.lancaster.ac.uk/social-futures/about-us/, consultado a 18.02.2020.
} 
geo-históricas bem definidas. Como dito anteriormente, as ideias de fluxo (Appadurai, 1990; Hannerz, 1992; Lash e Urry, 1994), rede (Castells, 1999), fluidez (Bauman, 1999), conexão (Jenkins et al., 2015), circulação (Appadurai, 1986; Harvey, 1992), rota (Kaplan, 1996; Clifford, 1997), malha (Ingold, 2007) e mobilidade (Bauman, 1999; Urry, 2000; Cresswell, 2006) passaram a ser acionadas como chaves interpretativas em diagnósticos elaborados pelas ciências humanas e sociais, elas próprias cada vez mais internacionalizadas. A contrapelo do vocabulário hegemônico que balizou o processo de institucionalização da sociologia, tal léxico aponta para um mundo social de fluxos relacionais e interdependentes, destituído, portanto, de estabilidade ontológica.

Em Sociology beyond Societies (2000), Urry recupera essa trajetória de implosão da sinonímia entre social, sociedade e Estado-nação. Porém, diante da complexidade associativa dos fenômenos, não se trata de simplesmente evitar a naturalização da escala do nacional. Cabe repensar, enfatiza Urry, a potência heurística do conceito mesmo de sociedade em um mundo no qual as relações sociais já não se dão exclusivamente face a face, mas são cultivadas a partir de diferentes escalas, inclusive de "mobilidades miniaturizadas" propagadoras de uma "intimidade à distância" (Elliot e Urry, 2010).

Anterior às novas relações globais e seus "espaços fractais", a noção de rede ganha peso nesse processo de suspensão cognitiva, graças à afinidade eletiva, por assim dizer, entre o conceito e a natureza das conexões emergentes: desprovidas de um único centro de comando, ricas em comunicações horizontais, atravessadas por fluxos sobrepostos, afeitas a fronteiras territoriais pervasivas, em grande medida simultâneas e instantâneas, não-lineares e não planejadas. A despeito de ser um conceito metafórico, um topos ou motivo utilizado em vários ramos da ciência, na teoria social a "rede" caracteriza o agregado de relações entre entidades conectadas que "viajam através e entre diversas paisagens [scapes], as quais se intervencionam de maneira complexa, imprevisível e sob formas da compressão espaço-temporal" (Urry, 2003: 72). ${ }^{3}$ As "redes sociais", por sua vez, referem-se ao conjunto esparso de relações de convivência, aos laços ou conexões interpessoais. Se as tecnologias de transporte e de comunicação, desde sua origem, reconfiguram o modo como as pessoas se conectam aos lugares e entre si, impactando a definição dos papéis sociais, é preciso pensar o que garante a preservação dessas relações sociais de convivência "com outros ausentes" (Elliott e Urry, 2010).

\footnotetext{
${ }^{3} \mathrm{Na}$ ausência de versão em português, todas as citações ao longo do texto são da nossa responsabilidade.
} 
A influência recíproca entre ideia e realidade descrita incrementa, porém, uma substancialização indesejada do fenômeno das redes. Ciente desse risco, Urry $(2000,2007)$ argumenta contra o "fetichismo do movimento" e recusa tratar a rede como fator explicativo absoluto, erro em que por vezes incorreria o autor da trilogia $A$ era da informação:

a obra-prima de Castells carece de um conjunto de conceitos interligados que permitiria uma compreensão sistemática de fenômenos muito diversos. O global é tido como certo e [...] ele espera que o termo 'rede' cumpra a maior parte do trabalho teórico no argumento. [...] Esse conceito encobre e confunde fenômenos em rede muito diferentes entre si. (Urry, 2003: 11-12)

Tanto em Sociology beyond Societies quanto em Global Complexity, Urry explicita certa desconfiança em relação à rede como operador analítico. Posteriormente, a linguagem das redes é por ele melhor qualificada com o conceito de capital de rede, como veremos na terceira seção. Antes, é preciso examinar como o diálogo com a teoria da complexidade o inspirou a pensar o mundo social como um composto de sistemas sobrepostos que capacitam ou detêm a mobilidade de pessoas, coisas, informações e imagens.

\section{Sistemas complexos de mobilidades múltiplas}

Como já amplamente mapeado, a ideia de complexidade emerge como resultado da reflexão em torno dos chamados sistemas dinâmicos nas ciências duras. Em sistemas feitos de bilhões de iterações, pequenas modificações em uma única operação podem, por conta de reações em cadeia, gerar efeitos desproporcionais e imprevisíveis, sendo "complexidade" o termo utilizado para descrever essas consequências não-lineares, nem completamente ordenadas, nem anárquicas. Não cabe aqui uma longa apreciação desses modelos, porém, é importante atentar que eles pressupõem uma ligação estreita entre os padrões de larga escala e a microdinâmica dos acontecimentos. De unidades absolutas e autônomas, espaço e tempo passam a ser tratados como qualidades contextuais, coprodutores dos processos em que estão implicados. Essas premissas impactarão os teóricos das ciências sociais e humanas, interessados nos paradoxos dos processos globais que ultrapassam as fronteiras das "sociedades", em descrever seus modos de emergência e a alta imprevisibilidade de seus efeitos.

Desde fins dos anos 1980, Urry defendia os ganhos epistêmicos de se observar as complexidades que perfazem os arranjos socioespaciais, econômicos e culturais de diferentes localidades, o que nos levaria a superar tanto a mera descrição idiográfica ou "estudos de caso", quanto a tendência 
de raciocínio nomotético em torno das relações necessárias e da superação dialética (cf. Bærenholdt, 2019). Em Global Complexity e em várias de suas obras posteriores - com destaque para a tetralogia pós-carbono ${ }^{4}-\mathrm{o}$ autor explicita esse flerte com a ideia de "desordem ordenada": eventos singulares ou "pequenas causas, em certas ocasiões, produzem grandes efeitos e vice-versa" (Urry, 2006: 113). Não há separação definitiva entre causas e efeitos, pois "tudo implica tudo", à semelhança de um holograma, cujas partes contêm o todo (Urry, 2003: 51).

Com propriedades similares às da metáfora das redes, os sistemas complexos são necessariamente dinâmicos, não-lineares e auto-organizados por feedbacks negativos e positivos, sendo os últimos responsáveis por distanciá-los do equilíbrio. Com efeito, o mobilities turn fala em "sistemas globais complexos marcados por conexões em rede" (Sheller, 2017: 632). Os sistemas de mobilidade estariam também estruturados de maneira complexa e dinâmica, como "constelações específicas de mobilidades desiguais" (ibidem: 9; ver Cresswell, 2006). Neles, como se pode ver na Tabela 1, cinco tipos básicos de mobilidade se entrelaçam, se intercalam e se sobrepõem.

TABELA 1 - Os cinco tipos de mobilidades interdependentes

(i) Mobilidade corporal de pessoas: deslocamento, cotidiano ou esporádico, de corpos com diferentes capacidades físico-sociais e motivações;

(ii) Movimento físico de objetos: circulação de coisas materiais, quer como mercadorias, quer como elementos da dádiva;

(iii) Mobilidade imaginativa: circulação, através de diferentes mídias, de imagens diversas e em disputa; capacidade de se projetar em outro lugar através dessas imagens e memórias; construção de paisagens sensoriais, que envolvem não apenas a visão, mas tato, olfato, audição e paladar;

(iv) Mobilidade virtual: circulação, em tempo real, proporcionada por tecnologias que diluem distâncias geográficas; navegar na Internet ou no ciberespaço;

(v) Mobilidade comunicativa: circulação de mensagens entre pessoas via dispositivos de comunicação - carta, telefone, e-mail, aplicativo de mensagens instantâneas, etc.

Fonte: Urry (2000, 2004, 2007); Larsen et al. (2006); Elliott e Urry (2010).

Não existe movimento puro, ou em si, apenas movimento localizado em uma das formas pelas quais instituições e práticas sociais são organizadas

${ }^{4}$ Climate Change and Society (2011), Societies beyond Oil (2013), Offshoring (2014) e What Is the Future? (2016) foram os últimos livros monográficos de Urry e marcaram sua guinada em direção a uma "sociologia pública", comprometida com a crítica ao capitalismo e seus excessos. 
no espaço e no tempo. Isso traz, pelo menos, duas implicações importantes. Primeiro, impõe repensar a relação entre as entidades não-humanas e os humanos, sendo estes tomados como seres psicobiológicos, expressivos, socializados e capazes de se mover por "caminhos" e de construir "pontes" de forma planejada e intencional (Simmel, 1996). Em confluência com os conceitos da teoria do ator-rede (TAR) (ver Latour, 1994; Law, 1994), Urry considera que "as sociedades são necessariamente híbridas" (Urry, 2000: 15) e, por essa razão, não existem nem relações sociais sem sistemas de entidades não-humanas a elas agregadas, nem sociedades humanas dominando uma natureza que lhe é apartada. Segundo, torna-se impositivo observar não só o movimento, mas os fixos e as fricções, pausas e turbulências. Daí a tríade - mobilidades, imobilidades e ancoradouros - presente no título do primeiro editorial do Mobilities Journal (Hannam et al., 2006).

A noção de ancoradouro (moorings) coloca acento na dependência do movimento em relação aos fixos, às infraestruturas materiais que o sustentam, vigiam e retêm: estradas, aeroportos, redes de $w i-f$, cabos de fibra óptica, redes elétricas, mas também checkpoints, cancelas aduaneiras, etc. Por elas podem ou não passar transeuntes, aviões e mercadorias, mas também afetos, mensagens e imagens. Cabe tratar empiricamente os ancoradouros como "uma infraestrutura socioespacial de interação que é atravessada por circuitos de mobilidade que para ali convergem e, já transmutados, são projetados em várias direções" (Freire-Medeiros e Pereira da Silva, 2019: 82; ver Freire-Medeiros e Name, 2019). O complexo sociotécnico resultante é descrito, em cada caso, como um "sistema de mobilidades" que pressupõe performances alinhadas e produz subjetividades específicas.

Algo ou alguém só é móvel em relação aos outros e até os lugares aparentemente mais isolados possuem seus tipos e intensidades de fluxo. Tal afirmativa assume evidência empírica quando observamos os destinos das performances turísticas: para que possam ser places to play, por onde passam corpos, suvenires e capitais, tais localidades precisam estar antes, elas mesmas, em movimento - places in play -, uma circulação antecipada e dependente dos suportes característicos das mobilidades imaginativas e virtuais (Sheller e Urry, 2004; ver também Freire-Medeiros, 2013). Além de ter se beneficiado diretamente da sobreposição entre várias "invenções disruptivas" do século XIX, como as ferrovias e a fotografia, o turismo como sistema de mobilidades só vingou graças à institucionalização de toda uma gama

\footnotetext{
5 Esta definição, assim como muitas das ideias sobre as quais se ergue o NMP, é claramente devedora das formulações de George Simmel, para quem mobilidade, racionalidade e individualidade compartilhariam o estatuto de princípio geral da Modernidade.
} 
de leis (férias pagas, entre outras), serviços (a hospitalidade como mercadoria), infraestruturas (de transporte e de comunicação), protocolos estéticos (a apreciação da paisagem visual e, mais recentemente, da gastronomia), disposições comportamentais e corporais (para a viagem, o lazer e o consumo), discursos especializados e espacializados (sobretudo em torno do binarismo exótico versus familiar) e moralidades ("ethos hedonista"), que evidenciam e reforçam padrões de desigualdade entre corpos e territórios. Sob as lentes das mobilidades, portanto, perde sentido a compreensão convencional que polariza e dissocia tanto os usos do tempo e dos espaços - trabalho versus lazer - quanto as identidades dos entes em movimento - migrantes versus turistas -, uma vez que os fluxos se sobrepõem, se hierarquizam, se reforçam mutuamente enquanto sistemas complexos.

Obviamente essa característica sistêmica - agentes e objetos associados a infraestruturas materiais, serviços e imaginários - não diz respeito somente às mobilidades turísticas. O NMP tem investido esforços, por exemplo, no escrutínio do "sistema da automobilidade" (ver, entre outros, o dossiê "Automobilities", publicado em 2004 no periódico Theory, Culture and Society). Trata-se de um sistema "não-linear e auto-organizativo" (Urry, 2003: 69) constituído por materialidades infraestruturais - rodovias, postos de abastecimento de combustível, motéis de beira de estrada -, assim como por uma imensa variedade de produtos, tecnologias e signos gerados para servir ao híbrido carro-motorista - das regulações de trânsito às campanhas publicitárias (Dennis e Urry, 2009). No encadeamento provocado pelo triunfo do carro no século $\mathrm{xx}$, a vida social acabou aprisionada a todo um sistema que depende, em última instância, de uma fonte de energia não renovável (Urry, 2003: 72).

Ao vincular a circulação física ou geográfica à virtual ou imaginativa, o NMP busca compreender as interfaces entre a mobilidade relativa a distâncias socioeconômicas (movimento para cima e para baixo nas hierarquias de dinheiro, classe e status) e a mobilidade relativa a distâncias socioespaciais (movimento no eixo horizontal dos espaços virtual e/ou físico). É do que trata a próxima seção.

\section{Capital de rede: operacionalizando as desigualdades intrínsecas aos regimes de mobilidade}

Refugiados, migrantes, turistas, terroristas, mercadorias (lícitas e ilícitas), informações e fake news, assim como tudo mais que se desloca pelo espaço, são definidos por relações de poder inscritas nos regimes que regulam mobilidades e imobilidades, o direito e o dever de chegar, de habitar e de partir. Em diálogo com a ideia foucaultiana de "regimes de práticas", a noção 
de "regime de mobilidades" incorpora a dimensão propriamente política em um quadro conceitual de entendimento da experiência subjetiva de mobilidade vis-à-vis as práticas normativas e dispositivos de vigilância que definem o que e quem pode (ou não) circular da forma o mais previsível e ao menor custo possível (cf. Shamir, 2005; Adey, 2010; Glick Schiller e Salazar, 2013; Sheller, 2018).

Contra a narrativa da globalização como fluxo contínuo (flow) de finanças, ideias e pessoas, o regime de mobilidades a expõe em suas fricções constitutivas, próprias de um fluxo oscilante e inconstante (flux), com vias expressas para poucos e cancelas para muitos. É da natureza do regime de mobilidades, porém, embaçar essa dimensão política e ideologicamente enviesada de seu funcionamento. Suas tecnologias de identificação, mensuração e rastreamento, bem como as decisões sobre infraestruturas de mobilidade, que não só expressam, como também reproduzem relações de poder altamente desiguais (cf. Graham e Marvin, 2001), são apresentadas como questões de cunho meramente técnico. No caso das mobilidades turísticas, como argumentado em $O$ olhar do turista, essa opacidade tende a assumir tons ainda mais densos (Urry, 2001; Urry e Larsen, 2011). Tanto que concepções normativas, inscritas em dispositivos fundamentais ao funcionamento do turismo - passaportes, vistos, alfândegas -, muitas vezes nos passam despercebidas como fricções regulatórias que são.

"A prática do poder", argumenta Cresswell (2014: 111), "diz respeito, via de regra, à administração das fricções - tirânicas para uns e atenuadas para outros". Nem todos se movem com a mesma velocidade ou podem ativar rotas confiáveis e seguras; poucos têm capacidade de gerenciar seus deslocamentos, físicos ou virtuais, de acordo com seus interesses. Resgatam-se, na grade analítica das mobilidades, os clássicos problemas da exclusão social e da desigualdade de acesso:

A inclusão social é uma questão de ultrapassar as limitações espaciais em momentos específicos do tempo, de modo a obter acesso às redes informais do trabalho, do lazer, da amizade e da família. Existe um "ônus de mobilidade" para sustentar as redes sociais [...]. Ao relacionar as noções de sociedade em rede à análise de como o lazer, a família e a vida profissional se tornaram (em média) mais dispersos, mais extensos e menos sobrepostos, notei algumas implicações para o conceito e o discurso do “acesso". (Urry, 2007: 193-194)

Em diálogo com as noções consagradas de capital econômico e capital cultural, mas reconhecendo a limitação destas no entendimento dos arranjos sociais em que a ausência física é a regra e a mobilidade espacial, imperativo 
e valor, o NMP propõe o conceito de "capital de rede", definido como "a capacidade de gerar e sustentar relações sociais com pessoas não necessariamente próximas e que produzem benefícios emocionais, financeiros e práticos (embora isso envolva frequentemente vários objetos e tecnologias ou meios de construir redes [networking])" (Urry, 2007: 197).

Documentos (passaportes e vistos); recursos financeiros e qualificações adequadas (incluindo o domínio de idiomas estratégicos); admissão livre e contínua a redes geograficamente descontínuas; capacidades físico-motoras; pontos de informação e contato; acesso a aparelhos de comunicação e a lugares de encontro seguros (imunes a vírus de toda sorte); proximidade a infraestruturas de transporte; uso do tempo e de outros recursos para coordenar a vida em rede - em conjunto e de maneira interdependente, esses oito elementos seriam a matéria-prima do capital de rede e a moeda dos regimes de mobilidade (Urry, 2007: 197-198; Elliott e Urry, 2010: 10-11). Se o capital de rede diz respeito às "relações sociais de circulação" (Urry, 2007: 197), a riqueza que lhe é própria são as socialidades construídas por meio de conexões dispersas.

Urry nos convida a observar os cruzamentos entre as maneiras de se deslocar e os marcadores - raça, gênero, geração, classe, nacionalidade, etc. - que produzem friç̧ões, hierarquias e distinções nos fluxos. Analogamente às outras formas de capital, o capital de rede pode reforçar desigualdades, trazendo para primeiro plano tanto as geometrias de poder inscritas no movimento (Massey, 1993) quanto o seu caráter relacional e situacional: "As iniquidades sociais são, via de regra, espaciais, resultando de formas altamente desiguais de acesso a, ou efeito de, vários tipos de mobilidade" (Urry, 2000: 195).

O NMP se aproxima de versões do conceito de capital social que enfatizam o caráter em rede e vão além da dimensão da copresença física (cf. Bourdieu, 1980; Lin, 1999; Wellman, 2001). Dito isso, é preciso sublinhar que habitam o âmago do capital de rede os contatos pontuais, que tendem a ser "efêmeros", mas que podem também ser produtivos, "intensos, focados, rápidos e sobrecarregados" (Urry, 2007: 221). Contra versões da teoria do capital social que só veem confiança, apoio e cuidado nas interações geridas nas comunidades de vizinhança e estendidas no tempo, Urry percebe a positividade dos "laços fracos" (Granovetter, 1973) que resultam do enlace entre copresença física, telepresença, virtualidade e imaginação. As interações presenciais, resultado da nossa "compulsão por proximidade" (Boden e Molotch, 1994), não são preteridas, ao contrário: "a viagem e as comunicações são entrelaçadas uma na outra de forma complexa” (Larsen et al., 2006: 124), as tecnologias de transporte e de comunicação são "parceiras de viagem". Indo mais além, 
pode-se argumentar que as próprias fronteiras entre o físico e o digital foram embaralhadas, criando "espaços híbridos" permeados pelo uso de tecnologias móveis atentas à localização cambiante de seus usuários, e resultando em diferenciais de mobilidade e espaço (de Souza e Silva, 2006; de Souza e Silva e Frith, 2010).

Em jogo está a capacidade do sujeito de aumentar o networking, de circular em redes estratégicas para obtenção de conhecimento tácito e outras vantagens, e de intensificar mobilidades potencialmente geradoras de novas mobilidades. A ênfase do capital de rede recai sobre os aspectos subjetivos e interpessoais, sobre relações sociais estimuladas pelo caráter decisivo que as viagens - físicas, virtuais ou imaginativas - assumem. ${ }^{6}$ Parafraseando a formulação do autor de $O$ capital, "é necessário examinar as relações sociais que os meios de mobilidade possibilitam e não apenas a mudança de forma produzida pelas forças de mobilidade" (Urry, 2012: 27; itálicos nossos). Se, como afirma Marx (2014: 205), "o capital, como valor que valoriza a si mesmo [...] só pode ser compreendido como movimento, e não como coisa imóvel", o movimento de pessoas e coisas é precondição para a criação de mercadoria. Do mesmo modo, Urry problematiza a concepção de que o acúmulo indiferenciado de conexões seria suficiente para produzir riquezas. Em vez de "um atributo de sujeitos individuais", o capital de rede deve ser entendido como "um produto da relacionalidade dos indivíduos com outros e com as disponibilidades [affordances] do 'ambiente"' (Urry, 2007: 198). O poder teria menos a ver com o que você sabe ou possui, e mais com quem você conhece e se relaciona. Em suas palavras:

O conceito de capital de rede ressalta que as mobilidades subjacentes nada fazem por si mesmas. O fundamental são as consequências sociais dessas mobilidades, a saber, ser capaz de gerar e manter relações sociais com pessoas (e visitar lugares específicos) [...], formar e sustentar redes via de regra mantidas à distância. Assim, o capital de rede aponta para as relações sociais reais e potenciais que as mobilidades proporcionam. (Urry, 2012: 27)

Em Mobilities (Urry, 2007), obra na qual reflexões que vinham sendo gestadas nas décadas anteriores encontram-se sistematizadas sob a alcunha de new mobilities paradigm, Urry elenca quatro componentes limitadores do "acesso", começando pelo aspecto econômico que, relativo às divisões de classe, seria a maior das restrições à mobilidade. Vale lembrar a que sistema econômico o autor se refere: intensivo na circulação de dados,

\footnotetext{
${ }^{6}$ Visando sublinhar as consequências propriamente sociais das mobilidades, Urry optou pelo termo capital de rede, em vez de capital de mobilidade ou motilidade (ver Kaufmann, 2002).
} 
este exige dos indivíduos que sejam capazes de fomentar circuitos econômicos baseados em fluxos simbólicos e que deles participem como produtores e consumidores de símbolos. As possibilidades de participação são repartidas de maneira extremamente assimétrica - o acesso diferencial aos meios de transporte e às estruturas de dados e comunicação, concentradas em espaços privilegiados, racializados e generificados, é crucial para a inserção dos indivíduos nas redes de poder próprias aos universos valorizados da cultura e da economia eletrônica global. Os demais limitadores - físico, organizacional e temporal - estariam subsumidos na noção de capital de rede e ganhariam importância crescente no mundo globalmente conectado (Urry, 2007: 194). Em conjunto, aspectos físicos (percorrer distâncias, dirigir máquinas...), organizacionais (logística de acesso a estradas, redes de $w i-f$, banco de dados...) e temporais (transporte em horários regulares, disponibilidade de agenda...) confluem na criação de desigualdades não-econômicas, embora economicamente mediadas.

Com o advento da revolução digital, somos obrigados a lidar com objetos materiais e virtuais que intermediam nossas interações em diferentes escalas. Smartphones são considerados itens de sobrevivência justamente por nos permitir coordenar no tempo nossas múltiplas atividades profissionais e pessoais, agendar e reagendar compromissos, transformando a pontualidade moderna do relógio em "tempo negociado" (Elliott e Urry, 2010; ver a entrevista de Elliott para Freire-Medeiros e Lages, 2018: 305; ver também o conceito de "microcoordenação" de Ling e Yttri, 2002). Indivíduos com elevado capital de rede sabem coordenar com eficiência essa complexidade, no plano cotidiano, e fazer bom uso dos fluxos informacionais oferecidos pelos encontros, gerando assimetrias de oportunidades que reforçam, por sua vez, a competência inicial.

Será sempre difícil prever as prioridades dos excluídos da "boa vida móvel" por conta do (velho) dilema de que, uma vez disseminado, o raro e apropriado pelas elites cinéticas - a mais "nova" novidade - cede lugar a outros bens e signos. Ler as desigualdades apenas em termos da "acessibilidade" é ignorar que tudo o que é valorizado em um momento histórico está imerso em sistemas de mobilidades e é reconstruído pelos sistemas em movimento; alterações na infraestrutura de "acesso" reorganizam a hierarquia dos bens e isso, por sua vez, redefine o que compõe a "exclusão" (Urry, 2007: 192). Obviamente, qualquer reflexão a esse respeito, no contexto latino-americano, terá que levar em conta o caráter altamente desigual de acesso a, e distribuição de, certas infraestruturas vitais de mobilidade, que residem em espaços secretos e impenetráveis, não raro militarizados e apartados dos que mais precisam deles. 
Nesse sentido, e por tudo mais que foi dito até aqui, parece-nos pertinente inferir dois axiomas: (i) o social, com suas iniquidades estruturais, deve ser entendido a partir da multiplicidade dos fluxos que conformam comportamentos, necessidades e desejos em disputa; (ii) posto que a manutenção dos vínculos sociais requer, cada vez mais, comunicações e viagens intermitentes, diferentes tipos de mobilidades se sobrepõem e impactam radicalmente as desigualdades de oportunidade e acesso. É o que a Tabela 2 abaixo, ainda que inconclusiva, busca sintetizar:

\section{TABELA 2 - Modelo analítico do paradigma das mobilidades}

Mobilidades: deslocamentos de várias ordens; entrelaçamento de movimento (realizado, potencial ou impedido), representações (discursivas, imagéticas e simbólicas) e práticas (performances e normatividades); dependentes de ancoradouros e de infraestruturas materiais conectadas; sempre em disputa.

Entes em movimento: pessoas, objetos materiais, imagens, signos, símbolos, informação, conhecimento, bens culturais, tecnologias, capital, afetos, riscos, vírus, gostos, estilos, etc.

Redes: modo de organização das relações sociais sob a dispersão espaço-temporal, representado graficamente por linhas que interligam nódulos ou pontos; evidências empíricas relativizam a assertiva, bastante frequente, de que as redes são menos hierarquizadas do que outras formas organizacionais.

Redes sociais: vínculos construídos nas interações; pressupõem enlace entre presença e ausência, proximidade e distância; envolvem compartilhamento de afeto, apoio e conhecimento tácito.

Capital de rede: agregado de competências para gerar e sustentar relações à distância que produzam benefícios emocionais, financeiros e práticos; capacidade de coordenar interações por meio de mobilidades físicas e telemediadas; sobrepondo-se parcialmente ao capital social, inclui precondições de acesso que são escassas e distribuídas de forma desigual.

Sistemas de mobilidades: complexos em que se sobrepõem os cinco tipos de mobilidades, e nos quais se distribui agência entre pessoas, objetos, lugares, tecnologias, signos e infraestruturas para possibilitar mobilidade, pausa ou fixação.

Regimes de mobilidades: medidas econômicas, intervenções políticas, decisões gerenciais, discursos de várias ordens (da ciência ao marketing), enunciados normativos e dispositivos de controle que, em conjunto, enquadram e definem as práticas de movimento, assim como disputam os sentidos subjetivos das mobilidades.

Fonte: elaboração nossa.

\section{Conclusão}

Durante a escrita deste artigo, fomos surpreendidos pela suspensão da vida social como a conhecíamos. A pandemia de covid-19, proclamada no dia $11 \mathrm{de}$ março de 2020 pela Organização Mundial de Saúde, gerou consequências 
disruptivas no regime de mobilidades de centenas de países, muitos dos quais passaram a adotar medidas severas de controle dos deslocamentos físicos, incluindo o fechamento das fronteiras nacionais (para as pessoas estrangeiras, vale lembrar), ao mesmo tempo que passaram a incentivar as conexões telemediadas em várias esferas. Espaços e tempos do trabalho, do lazer e dos afetos foram redefinidos para conter a mobilidade indesejada do vírus e a velocidade de sua propagação.

Uma das consequências mais impactantes foi, sem dúvida, a diminuição do ritmo da vida urbana: mais de um quinto da população das grandes cidades esteve, em algum momento, sob lockdown ou confinamento. ${ }^{7}$ Se a cidade moderna, como construto mental e como realidade empírica, foi concebida e planejada para viabilizar, conduzir e controlar a mobilidade de pessoas, mercadorias e informações, não surpreende que nos cause um estranhamento radical, verdadeiro choque cognitivo, a imagem de avenidas, mundo afora, vazias de gente, sem trânsito de veículos, sem vida. Onde antes se tinha convergência de movimento e convívio, agora se exige isolamento e distância.

$\mathrm{Na}$ cosmologia de valores do capitalismo globalizado, a distância é, via de regra, qualificada em, pelo menos, dois sentidos. Por vezes, é aquilo que compromete o tempo de rotatividade entre produção e troca, a execução de tarefas coordenadas em equipe ou a qualidade daquilo que se transporta. Nesse sentido, é uma fricção que desacelera o movimento, um "atrito" a ser vencido pela velocidade e/ou pelo aperfeiçoamento a qualquer custo das tecnologias de comunicação e transporte (cf. Virilio, 1996 sobre a velocidade como valor). Mas, nos regimes de mobilidade que sustentam a ordem mundial, a distância também assume um sentido imperativo de organização hierárquica dos fluxos. Quando assim definida, é sinônimo de "separação" - pensemos na triagem entre turistas e terroristas, expatriados e indocumentados garantida por microdispositivos de escuta, cães farejadores, câmeras biométricas de reconhecimento facial e toda sorte de aparatos de vigilância que encontram nos aeroportos seu campo de experimentação, antes de se estilhaçar pelas cidades militarizadas.

A covid-19, enquanto um ponto de inflexão de escala planetária, obriga-nos a resgatar a "distância" do enquadramento tecnicista e militarizado que lhe veio sendo imposto. Isso significa, por um lado, abordá-la em termos de percepções, experiências e valores: como qualificamos o que nos é distante ou próximo; o que e quem nos compele a ultrapassar (ou preservar)

\footnotetext{
${ }^{7}$ Relatório feito a partir de dados de geolocalização apontou que os fluxos de transporte público haviam diminuído radicalmente no período inicial da pandemia, com uma queda de $86 \%$ em Nova Iorque, Estados Unidos e 78\% em São Paulo, Brasil. Cf. "COVID-19 - Relatórios de tendências de mobilidade - Apple”, consultado a 24.04.2020, em https://www.apple.com/covid19/mobility.
} 
os espaços que nos separam uns dos outros e daquilo que desejamos (ou tememos); em nome de quê e na companhia de quem é legítimo mover-se. Por outro lado, exige que iluminemos a economia política que dá sustentação à gramática dos distanciamentos: quem detém a propriedade sobre o ritmo com que percorremos as distâncias e quais são os jogos de poder que arbitram sobre a duração, o destino e a companhia dos nossos trajetos. Mais do que nunca, cabe atentar tanto para o quê exatamente está circulando quanto para o modo como está circulando e em relação a quê. A compreensão da fixidez, aderência material ou viscosidade dos sujeitos, objetos e relações nos ajuda a qualificar as distâncias como relacionais, relativas e definidas, não raro, em contextos de profunda assimetria.

Os super-ricos não hesitam, sempre que lhes é conveniente, em "alternar entre países e regiões, entre regimes fiscais e sistemas legais, enquanto vivem estilos de vida suntuosos e extraordinários” (Elliott e Urry, 2010: 67). Em situações de crise como a que estamos vivendo, os privilégios do capital de rede e da condição de alta mobilidade lhes garantem rotas de fuga rumo a paraísos sanitarizados. Diante do "medo da imobilidade", essas elites cinéticas globais podem optar por se mover ininterruptamente, anulando as distâncias que as separam de tudo que lhes traz segurança emocional e psicológica. Simultaneamente, detêm o privilégio de permanecer imóveis enquanto outros são obrigados a se deslocar pelas vias poluídas, em transportes coletivos infectados, enfrentando altos riscos de contaminação para servi-las. No caso brasileiro, se foram as elites cinéticas (membros do alto escalão de governo e turistas retornando de viagens internacionais) as responsáveis pela introdução do vírus entre nós, os mais vulneráveis são as pessoas com menos capital de rede, moradores das favelas e periferias para quem a distância segura garantida pelo autoconfinamento não é uma opção.

Já não resta qualquer dúvida de que nos tornamos "tão dependentes das mobilidades que, caso falhem, nossas vidas podem ser mergulhadas no caos" (Adey et al., 2014: 2). Sejam quais forem os legados sociotécnicos e humanos na vida social pós-covid-19, uma previsão pode ser feita: de tempos em tempos, ocorrerão perturbações no "discurso global da mobilidade irrestrita como modo de vida” (Hannam et al., 2006: 7). São os momentos de fricção, como este no qual estamos mergulhados, que trazem à consciência, da forma mais dramática, as assimetrias de poder que constituem as mobilidades em várias escalas. Mas não devemos esquecer, como nos mostra Anna Tsing, que nem tudo é impedimento e que há positividade na fricção - dela resultam engajamento e encontro, alianças e empréstimos, traduções e acomodações entre "tipos de conhecimento e culturas", que só podem emergir da "interconexão através da diferença" (Tsing, 2005: 5). 
Para uma "boa vida móvel", com fricções assim produtivas, há que se recusar a "normalidade" dos deslocamentos forçados e das remoções, minimizar a "imobilidade coagida" e favorecer as mobilidades que, a despeito das distâncias, realizam conversas, redes e encontros.

Revisto por Sofia Silva

\section{Referências bibliográficas}

Adey, Peter (2010), Mobility. London/New York: Routledge.

Adey, Peter; Bissell, David; Hannam, Kevin; Merriman, Peter; Sheller, Mimi (2014), "Introduction", in Peter Adey; David Bissell; Kevin Hannam; Peter Merriman; Mimi Sheller (orgs.), The Routledge Handbook of Mobilities. London: Routledge, 1-20. Appadurai, Arjun (org.) (1986), The Social Life of Things. Commodities in Cultural Perspective. Cambridge: Cambridge University Press.

Appadurai, Arjun (1990), "Disjuncture and Difference in the Global Cultural Economy", Theory, Culture \& Society, 7(2-3), 295-310.

Bærenholdt, Jørgen Ole (2019), "Going Places", in Ole B. Jensen; Sven Kesselring; Mimi Sheller (orgs.), Mobilities and Complexities. London: Routledge, 89-96.

Bauman, Zygmunt (1999), Globalização. As consequências humanas. Rio de Janeiro: Jorge Zahar. Tradução de Marcus Penchel.

Beck, Ulrich (2002), "The Cosmopolitan Society and its Enemies", Theory, Culture \& Society, 19(1-2), 17-44.

Boden, Diedre; Molotch, Harvey (1994), "The Compulsion to Proximity", in Roger Friedland; Deirdre Boden (orgs.), NowHere. Space, Time and Modernity. Berkeley: University of California Press, 257-286.

Bourdieu, Pierre (1980), "Le capital social: notes provisoires", Actes de la recherche en sciences sociales, 31(1), 2-3.

Büscher, Monika; Urry, John; Witchger, Katian (orgs.) (2011), Mobile Methods. London: Routledge.

Castells, Manuel (1999), A sociedade em rede. São Paulo: Paz e Terra.

Clifford, James (1997), Routes. Travel and Translation in the Late Twentieth Century. Cambridge, MA: Harvard University Press.

Cresswell, Tim (2006), On the Move. Mobility in the Modern Western World. London: Routledge.

Cresswell, Tim (2014), "Friction" in Peter Adey; David Bissell; Kevin Hannam; Peter Merriman; Mimi Sheller (orgs.), The Routledge Handbook of Mobilities. London: Routledge, 107-115.

de Souza e Silva, Adriana (2006), "From Cyber to Hybrid: Mobile Technologies as Interfaces of Hybrid Spaces”, Space and Culture, 9(3), 261-278. 
de Souza e Silva, Adriana; Frith, Jordan (2010), "Locative Mobile Social Networks: Mapping Communication and Location in Urban Spaces”, Mobilities, 5(4), 485-505. Dennis, Kingsley; Urry, John (2009), After the Car. Cambridge: Polity.

Elias, Norbert (1994), O processo civilizador. Vol. 1 - Uma história dos costumes. Rio de Janeiro: Jorge Zahar.

Elliott, Anthony; Urry, John (2010), Mobile Lives. London: Routledge.

Freire-Medeiros, Bianca (2013), Touring Poverty. London: Routledge.

Freire-Medeiros, Bianca; Lages, Mauricio Piatti (2018), "Mobilidades, espaços e identidades na economia eletrônica global: entrevista com Anthony Elliott", Tempo Social, 30(2), 301-316.

Freire-Medeiros, Bianca; Telles, Vera da Silva; Allis, Thiago (2018), "Apresentação: por uma teoria social on the move", Tempo Social, 30(2), 1-16.

Freire-Medeiros, Bianca; Name, Leo (2019), "Epistemologia da laje”, Tempo Social, 31(1), 153-172.

Freire-Medeiros, Bianca; Pereira da Silva, Nathalia (2019), "Um passeio além do temp(l)o: mobilidades, mídia e memória no Jardim Bíblico do Templo de Salomão”, in Edson Farias; Salete Nery (orgs.), Durações e redes de fluxos no cenário cultural contemporâneo. Jundiaí: Paco e Littera, 79-102.

Glick Schiller, Nina; Salazar, Noel B. (2013), "Regimes of Mobility across the Globe”, Journal of Ethnic and Migration Studies, 39(2), 183-200.

Graham, Stephen; Marvin, Simon (2001), Splintering Urbanism. Networked Infrastructures, Technological Mobilities and the Urban Condition. London: Routledge.

Granovetter, Mark S. (1973), "The Strength of Weak Ties”, American Journal of Sociology, 78(6), 1360-1380.

Gregory, Derek; Urry, John (orgs.) (1985), Social Relations and Spatial Structures. London: Palgrave Macmillan.

Hall, Stuart (1997), "The Local and the Global: Globalization and Ethnicity", in Anthony King (org.), Culture, Globalization, and the World-System. Contemporary Conditions for the Representation of Identity. Minneapolis: University of Minnesota Press, 19-40.

Hannam, Kevin; Sheller, Mimi; Urry, John (2006), "Mobilities, Immobilities and Moorings", Mobilities, 1(1), 1-22.

Hannerz, Ulf (1992), Cultural Complexity. Studies in the Social Organization of Meaning. New York: Columbia University Press.

Harvey, David (1992), Condição pós-moderna: criando valor e significado por meio da mídia propagável. Rio de Janeiro: Edições Loyola.

Ingold, Tim (2007), Lines. A Brief History. London: Routledge.

Jenkins, Henry; Green, Joshua; Ford, Saml (2015), Cultura da conexão. Criando valor e significado por meio da mídia propagável. São Paulo: Aleph. Tradução de Patricia Arnaud.

Kaplan, Caren (1996), Questions of Travel. Postmodern Discourses of Displacement. Durham/London: Duke University Press. 
Kaufmann, Vincent (2002), Re-Thinking Mobility. Contemporary Sociology. Aldershot: Ashgate.

King, Anthony (1984), The Bungalow. The Production of a Global Culture. New York: Routledge.

Larsen, Jonas; Urry, John; Axhausen, Kay (2006), Mobilities, Networks, Geographies. Hampshire: Ashgate.

Lash, Scott; Urry, John (1987), The End of Organised Capitalism. Madison: UWP.

Lash, Scott; Urry, John (1994), Economies of Signs \& Space. London: Sage.

Latour, Bruno (1994), Jamais fomos modernos. Ensaio de antropologia simétrica. Rio de Janeiro: Editora 34. Tradução de Carlos Irineu da Costa.

Law, John (1994), Organizing Modernity. Oxford: Blackwell.

Lin, Nan (1999), "Building a Network Theory of Social Capital”, Connections, 22(1), 28-51.

Ling, Richard; Yttri, Birgitte (2002), "Hyper-Coordination via Mobile Phones in Norway", in James E. Katz; Mark Aakhus (orgs.), Perpetual Contact. Mobile Communication, Private Talk, Public Performance. Cambridge: Cambridge University Press, 139-169.

Marcus, George E. (1995), "Ethnography in/of the World System: The Emergence of Multi-Sited Ethnography”, Annual Review of Anthropology, 24(1), 95-117.

Marx, Karl (2014), O capital, livro II. São Paulo: Boitempo. Tradução de Rubens Enderle. Massey, Doreen (1993), "Power Geometry and a Progressive Sense of Place", in Jon Bird; Barry Curtis; Tim Putnam; George Robertson; Lisa Tickner (orgs.), Mapping the Futures. Local Cultures, Global Change. London: Routledge, 60-70.

Nogueira, Maria Alice; Moraes, Camila dos Santos (orgs.) (2020), Brazilian Mobilities. London: Routledge.

Ortiz, Renato (1994), Mundialização e cultura. São Paulo: Brasiliense.

Revill, George (2014), "Histories”, in Peter Adey; David Bissell; Kevin Hannam; Peter Merriman; Mimi Sheller (orgs.), The Routledge Handbook of Mobilities. London: Routledge, 506-516.

Santos, Boaventura de Sousa (org.) (2001), Globalização: fatalidade ou utopia? Porto: Edições Afrontamento.

Sassen, Saskia (1991), The Global City. Princeton: Princeton University Press.

Shamir, Ronen (2005), "Without Borders? Notes on Globalization as a Mobility Regime", Sociological Theory, 23(2), 197-217.

Sheller, Mimi (2014), “Sociology After the Mobilities Turn”, in Peter Adey; David Bissell;

Kevin Hannam; Peter Merriman; Mimi Sheller (orgs.), The Routledge Handbook of Mobilities. London: Routledge, 45-54.

Sheller, Mimi (2017), "From Spatial Turn to Mobilities Turn”, Current Sociology, 65(4), 623-639.

Sheller, Mimi (2018), Mobility Justice. The Politics of Movement in an Age of Extremes. London: Verso. 
Sheller, Mimi; Urry, John (2004), Tourism Mobilities. Places to Play, Places in Play. London: Routledge.

Sheller, Mimi; Urry, John (2006), “The New Mobilities Paradigm”, Environment and Planning A, 38(2), 207-226.

Simmel, Georg (1996), "A ponte e a porta”, Revista Política \& Trabalho, 12, 11-15. Tradução de Simone Maldonado.

Singh, Dhan Zunino; Giucci, Guillermo; Jirón, Paola (orgs.) (2017), Términos clave para los estudios de movilidad en América Latina. Buenos Aires: Editorial Biblos.

Tsing, Anna (2005), Friction. An Ethnography of Global Connection. Princeton: Princeton University Press.

Urry, John (2000), Sociology Beyond Societies. Mobilities for the Twenty-First Century. London: Routledge.

Urry, John (2001), O olhar do turista. Lazer e viagens nas sociedades contemporâneas. São Paulo: Studio Nobel/SESC. Tradução de Carlos Eugênio Marcondes de Moura [orig. 1990].

Urry, John (2003), Global Complexity. Cambridge: Polity Press.

Urry, John (2004), “Connections”, Environment and Planning D, 22(1), 27-37.

Urry, John (2006), “Complexity”, Theory, Culture \& Society, 23(2-3), 111-117.

Urry, John (2007), Mobilities. Cambridge: Polity Press.

Urry, John (2011), Climate Change and Society. Cambridge: Polity Press.

Urry, John (2012), "Social Networks, Mobile Lives and Social Inequalities”, Journal of Transport Geography, 21, 24-30.

Urry, John (2013), Societies beyond Oil. Oil Dregs and Social Futures. London: Zed Books. Urry, John (2014), Offshoring. Cambridge: Polity Press.

Urry, John (2016), What Is the Future? Cambridge: Polity Press.

Urry, John; Larsen, Jonas (2011), The Tourist Gaze 3.0. London: Sage.

Virilio, Paul (1996), Velocidade e política. São Paulo: Estação Liberdade. Tradução de Celso Mauro Paciornik.

Wellman, Barry (2001), "Physical Place and Cyberplace: The Rise of Personalized Networking”, International Journal of Urban and Regional Research, 25(2), 227-252.

Wimmer, Andreas; Glick Schiller, Nina (2002), "Methodological Nationalism and Beyond: Nation-State Building, Migration and the Social Sciences”, Global Networks, 2(4), 301-334. 
Artigo recebido a 03.06.2020

Aprovado para publicação a 10.08.2020

\section{Bianca Freire-Medeiros}

Programa de Pós-graduação em Sociologia, Departamento de Sociologia, Faculdade de Filosofia, Letras e Ciências Humanas, Universidade de São Paulo Avenida Prof. Luciano Gualberto, 315 - sala 1057, Prédio de Filosofia e Ciências Sociais, Cidade Universitária - Butantã, 05508-010 São Paulo, Brasil

Contacto: bfreiremedeiros@gmail.com

ORCID: https://orcid.org/0000-0003-3121-7897

\section{Mauricio Piatti Lages}

Doutorando do Departamento de Sociologia da Universidade de São Paulo | Bolsista da Fundação de Amparo à Pesquisa do Estado de São Paulo (FAPESP)

Avenida Prof. Luciano Gualberto, 315 - sala 1057, Prédio de Filosofia e Ciências Sociais, Cidade Universitária - Butantã, 05508-010 São Paulo, Brasil

Contacto: murucopl@hotmail.com

ORCID: https://orcid.org/0000-0001-5359-6571

\section{The Mobilities Turn: \\ Flows, Fixities and Frictions}

At least since the 2000s, we have witnessed the effort to understand, in multiple disciplinary fields and from different empirical realities, the complexity of the interdependent movement of people and capitals, images and goods. But when static categories and sedentary methodologies are denied, how can we avoid the substitution of what is proper to the Nation-State with the conceptual imprecision of "liquids" or with the romanticization of nomadism? How can regulatory practices and inequalities be incorporated into the discussion of mobility? What, in short, would be the theoretical status of mobility - and immobility - in social theory? This article describes some of the analytical solutions proposed by the "mobility turn", taking the contributions of its main reference,

\section{Le tournant des mobilités : fluxes, fixités et frictions}

Au moins depuis les années 2000, nous avons été témoins de l'effort, dans différents domaines disciplinaires et à partir de diverses réalités empiriques, pour comprendre la complexité de la circulation interdépendante des personnes et des capitaux, des biens et des images. Mais comment éviter, dans le déni des catégories statiques et des méthodologies sédentaires, la substitution de ce qui est approprié à l'État-nation par l'inexactitude conceptuelle des « liquides » ou la romantisation du nomadisme? Comment pouvons-nous intégrer, dans la discussion sur la mobilité, les pratiques réglementaires et les inégalités qui la structurent ? Quel serait, en bref, le statut théorique des mobilités - et immobilités - au sein de la théorie sociale ? Nous décrivons dans cet article quelques-unes 
the British sociologist John Urry, as its guiding principle.

Keywords: epistemology of the social sciences; epistemic change; social theory. des solutions analytiques proposées par le «tournant des mobilités », prenant comme fil conducteur les contributions de celui qui est reconnu comme son principal articulateur, le sociologue britannique John Urry.

Mots-clés: changement épistémique; épistémologie des sciences sociales; théorie sociale. 